\title{
5. Workshop report: mental representations and discrete choice behaviour: state-of-the-art and avenues for future research
}

Benedict G.C. Dellaert, Theo Arentze, Caspar G. Chorus, Harmen Oppewal and Geert Wets

\section{INTRODUCTION}

This chapter considers the measurement and modelling of mental representations underlying individuals' choice behaviour. The premise of the study of such mental representations (MRs) is that an individual's mental construction of a choice problem plays an important role in preference formation, especially in the context of the complex choice situations such as involved in activity-travel behaviour. The measurement of MRs is a key problem. It requires the availability of a cognitive mapping method that can take specific decision task characteristics into account. Existing cognitive mapping methods, such as proposed in Kearney and Kaplan (1997), Eden and Ackermann (1998), and Christensen and Olsen (2002), cannot readily be used for this purpose. These traditional cognitive mapping techniques focus on knowledge and values not related to a specific situation or decision task. In contrast, MRs underlying decision making concern specific cognitions that are triggered by a given decision problem and should allow the decision maker (DM) to assess outcomes of actions in terms of needs that are activated in the decision situation.

To model and measure such cognitions, recently new methodology has been developed based on the concept of active causal networks. Specifically, Arentze et al. (2008) proposed the decision network (DN) as a suitable formalism. A DN is an extension of a Bayesian Network (BN). It links a $\mathrm{BN}$ for making inferences with decision variables and utility nodes representing choice options and preferences of a decision maker 
(Schachter 1986). A DN includes all knowledge needed to assess and evaluate consequences of actions and in that sense is a sufficient model. To measure MRs based on this formalism, the authors developed an interview method, called CNET (Causal Network Elicitation Technique) (Arentze et al., 2008). The interview method has been tested and applied to collect data on MRs (Dellaert et al. 2008). In subsequent work several new versions of the technique have been developed involving elaborations and adaptations that have automated and streamlined the processes (e.g., Horeni et al. 2010; Kusumastuti et al. 2010, 2011). These applications showed how the measurement method can reveal shifts in MRs induced by variations in situational variables and showed its scalability to relatively large samples.

The purpose of this chapter is to review and illustrate this relatively new field of research, discuss its potentials for discrete-choice modelling and identify promising avenues of future research. First, we briefly introduce basic concepts and methods, then give an illustration of an application and conclude with a discussion of potentials, challenges and avenues for future research.

\section{MENTAL REPRESENTATION THEORY: BASIC CONCEPTS AND METHODS}

The model of MRs proposed by Arentze et al. (2008) and Dellaert et al. (2008) draws on two areas of research: mental model theory of human cognition (Johnson-Laird 1983, 2001; Johnson-Laird and Byrne 1991) and formalisms of causal networks and causal reasoning in BNs and DNs (Pearl 1988; Schachter 1986; Spiegelhalter et al. 1993; Neapolitan 1990), although it should be noted there are also some similarities with meansend chain theory (Gutman 1982). Based on mental model theory we first identify the role MRs play in decision making. We then show how MRs can be modelled using the DN formalisms as a framework and how they can be used to evaluate actions.

When faced with a decision task, such as planning activities for a day or a trip, individuals need to activate or construct a MR of the system (i.e. of the environment and decision alternatives) they are interacting with. Different components can be distinguished in the MR. The situation refers to the system that is outside the control of the DM but does have an influence on outcomes of actions. Decision alternatives represent the possible states of the system that result from the DM taking action. The causal network is the component of the MR that is activated in a given situation and that allows the DM to assess the possible outcomes of actions. Individual needs arise from basic values, or desirable end-states, that the 
individual seeks, such as safety, social acceptance, or accomplishment (Gutman 1982). These values are relatively stable across situations. Utility weights are values that the individual assigns to possible states of the system given the needs activated in the situation.

As an example, consider a individual faced with the planning of a business trip. Assume that the location of the business activity is known at the moment of planning and that this location is some place outside the centre area of a big city. The location of the hotel, the length of stay and the transport mode of the trip are yet to be decided. The individual cannot immediately establish the ultimate utilities of choices, as outcomes depend on conditions that are hard to predict (i.e., the weather) or are influenced by other decisions (e.g., total costs depend on mode as well as accommodation choice). The desired time efficiency, cost efficiency and the possibility to enjoy oneself are activated needs of the individual that determine how he or she frames and evaluates outcomes. The causal network represents the individual's activated knowledge about transport systems, hotels, cities, climates, the own household, the work environment, etc., needed to assess the likelihood of outcomes on relevant variables. The decision problem is complex because the choices are interdependent (e.g., the preferred length of stay depends on the hotel location and transport mode) and the outcomes are dependent on uncertain elements of the situation (e.g., the weather, availability of hotels, etc.).

A crucial feature of the proposed conceptualization is that an MR is tailored to the task and situational setting at hand. Task and situational variables generally determine which variables of a system are given or are free to choose, which needs are activated and what the consequences of certain actions are. As a consequence, different situations and tasks tend to trigger different selections of variables and causal links. In the business trip example the activated needs and causal links will depend strongly on situational variables such as the length of the trip and whether or not other individuals are joining (e.g., the individual's spouse).

Furthermore for an MR to be active, it needs to be held in working memory. This implies that the amount of information that can be activated is limited by the capacity of working memory. As a result of the situation-dependency and limited capacity of the MR a change in situational setting may cause a significant change in the MR, which a DM would experience as a cognitive switch, that is, a qualitative change in the view on the problem. For instance, in the city-trip example, if the spouse would be joining instead of staying home, the MR of the DM may switch to viewing the problem in terms of a quite different set of variables, resulting for example in a higher salience of entertainment and accommodation variables. 
The nature of the MR, comprising an activation of decision alternatives, causal knowledge and value judgments, suggests that a $\mathrm{DN}$ is a suitable formalism to model an MR. The core part of a DN - the BN - consists of two components: (i) a (directed acyclic) graph, with nodes representing stochastic variables and directed arcs representing dependencies between the variables, and (ii) conditional probabilities for each node describing the dependency relationships captured by the graph (Pearl 1988). As an extension of a $\mathrm{BN}$, a $\mathrm{DN}$ furthermore represents the decision options available to the DM as well as nodes representing the utilities he or she associates with the outcomes. Algorithms for probability propagation across the network provide a mechanism for probabilistic reasoning and deriving expected utilities of decision options in these networks. This means that beliefs about likelihood of outcomes and expected utilities of decision options can be determined for any given configuration of evidence (i.e., data) about the variables.

To describe the causal network in more detail we make a distinction between situational, decision and outcome variables. Decision variables represent those elements of the system that the individual (the decision maker) is free to choose. Outcome variables represent consequences of decisions on relevant dimensions. Situational variables differ from outcome variables in that they are not directly or indirectly influenced by decisions. Within the category of outcome variables, we make a further distinction between attribute and benefit variables (Gutman 1982). Attribute variables relate to states of the system that are directly observable, whereas benefit variables describe outcomes on a more abstract level standing closer to need dimensions of the individual. Outcome variables can take a position somewhere between concrete and abstract, suggesting the MR should allow for a continuum of levels of abstraction from observable states. Figure 5.1 shows a DN model of the MR related to the earlier business trip example. The nodes are arranged in layers where the first layer represents the decision variables, the second layer includes the attribute variables and the third layer consists of benefit variables.

The MR further consists of conditional probabilities and conditional utilities. Conditional probabilities represent the detailed knowledge needed to use the network for reasoning. In general, the probability parameters allow the respondent to assess outcomes on attribute and benefit variables for each configuration of decision and situational variables assumed in a mental simulation. Beliefs regarding outcomes are uncertain if the states of situation variables or the involved inferences are uncertain.

Conditional utilities define how a respondent evaluates particular configurations of the system. In a DN, utility variables are incorporated as separate nodes in the network. Accordingly, an MR includes a utility 


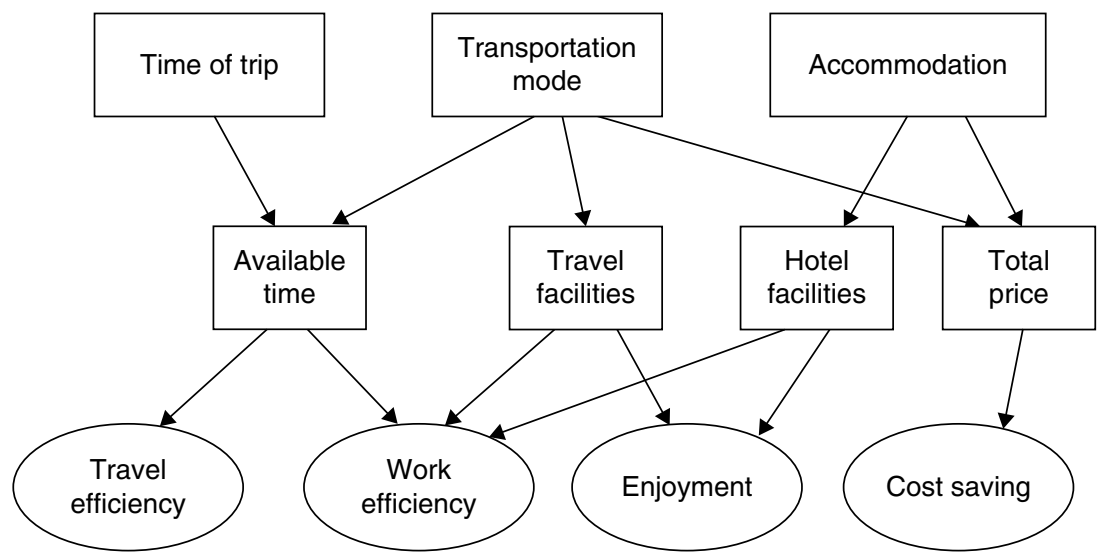

Figure 5.1 Example of an individual's mental representation

variable for each benefit variable and a link between the utility and the benefit representing their dependency relationship (see Figure 5.1). The range of utility values across outcomes of a benefit variable captures the relative importance of a benefit variable. The larger this range the more important the benefit variable is and vice versa. In the example, the range of the utilities may vary across budget, efficiency and enjoyment variables indicating that outcomes related to these benefits are not necessarily considered equally important.

Probability and utility parameters represent the knowledge needed to evaluate possible courses of actions, where 'action' refers to a particular configuration of decision variables. To the extent that situational conditions or inferences are uncertain, the outcomes of actions on benefit variables are uncertain. Probabilities propagate across the links of the network and are accumulated at the benefit nodes, which constitute the end points of reasoning chains. The expected utility accumulated at a benefit variable is the weighted sum of utilities across outcomes, using the probabilities as weights. The overall expected utility for a scenario equals the sum of expected utilities across the benefit variables.

In the face of uncertainty, individuals may use different decision rules depending on their attitude towards risk. Examples of decision rules include 'choose the action that maximizes the utility of the worst outcome' (risk aversive), 'maximize the utility of the best outcome' (risk taking) and 'maximize the expected value across outcomes' (risk neutral). The decision rule is not part of the MR. Thus, the proposed formalism supports decision making based on different possible decision processes or styles a DM may use. 


\section{MEASURING MENTAL REPRESENTATIONS}

It follows from the foregoing that the measurement of an MR involves observing a causal network, conditional probabilities, and conditional utilities. In this section we describe the CNET method, which is designed to acquire this information in a face-to-face interview with a respondent who is presented with a choice problem. In later applications the approach was automated to allow for more flexible large-scale data collection. For clarity, we here describe the original non-automated approach. The method is somewhat similar to the 'laddering' approach (Reynolds and Gutman 1988) associated with means-end theory but it adopts a more structured and systematic approach. For example it includes a predefined list of attributes (resembling 'hard laddering', Russell et al. 2004). The method is illustrated with a case application in the next section but in general starts with generating an initial list of attribute, benefit and situational variables for the choice problem under concern. This list is brought to the interview but is not shown to the respondent, as this might induce variables that are not part of the MR. Rather the interviewer asks openended questions and uses the lists to identify responses in terms of these variables. The list does not need to be exhaustive. If a mentioned variable does not match with any of the items in the list, the interviewer adds it to the list. Since decision variables are defined in the task description, they are not asked but presented to the respondent. However, the priority order of the decision variables is considered part of subjective representation and, hence, part of the information the interview should reveal.

The interview proceeds through the following steps. The aim of the first step is to determine the order of decisions. The decision variables given by the task are written on cards which are placed in a random arrangement in front of the respondent. The interviewer asks the respondent to place the cards in the sequence in which he/she would prefer to make the decisions. The order of decisions plays a role in the elicitation of the causal network in the next step.

The aim of the second step is to reveal the causal network. The interviewer considers the decision variables in the order indicated by the respondent and repeats the following procedure for each decision variable. The interviewer explains the choice alternatives involved and asks 'What are your considerations when faced with these alternatives?' The interviewer tries to identify from the list of pre-defined variables the one that corresponds to the answer given. If none of the variables matches, a new variable is defined and added to the list. The interviewer next asks the respondent whether the variable is correct and determines whether the respondent believes it is causally linked to the decision variable or not. 
If the identified variable is an attribute, the interviewer proceeds by asking 'Why is this consideration important for the decision?' The answer to this question should lead to the identification of a benefit variable and, in that case, no further 'why' questions are needed. If the variable identified is a benefit variable, the interviewer proceeds by asking ' $\mathrm{How}$ is this variable influenced by other factors?' This question may lead to identifying other situational or attribute variables. Depending on the nature of the variables, the interviewer establishes the relevant causal links and verifies these with the respondents if necessary.

After the first decision consideration is processed in this way, the interviewer prompts the respondent for other considerations by repeating the above questions until no further decision considerations are mentioned. The interviewer repeats the entire procedure for the next decision variable and so on until all decision variables have been processed. Note that the same consideration (attribute or benefit) may play a role in the context of different decisions. In that case the interviewer does not add a new variable, but rather establishes a link with the variable entered earlier.

Having recorded the structure of the network, the next steps are concerned with establishing the probability and utility parameters. First, the probabilities are considered. From top to bottom layer, the interviewer goes through the situational, attribute and benefit variables in the network, one by one. For each variable, the interviewer presents the possible state configurations of the parent variables and asks the respondent to indicate his/her assessment of the probabilities of outcomes on the variable under each condition. Finally, utilities can be measured, for example by means of a stated preference task. In this task, the respondent indicates his/her preference for each of a number of profiles presented. A profile represents a particular state configuration of all benefit variables included in the network. By asking respondents to judge complete profiles, they are forced to take the relative importance of each benefit variable into account. It is not necessary to have the respondent evaluate all profiles that can be formed as combinations of states of the benefit variables. A well-chosen fraction of a full factorial design generally suffices to estimate the underlying part-worth utilities. The interviewer presents the profiles one by one and asks the respondent to indicate on a $0-100$ scale how likely it is that he/she would implement a plan under the conditions stated.

In eliciting and recording the responses the interviewer takes care not to interfere with perceptions and knowledge of the respondent. By adhering to the protocol the interviewer aims to obtain a recorded MR that is as unambiguous, complete and internally consistent as possible. 


\section{EXAMPLE APPLICATION: LEISURE SHOPPING TRANSPORT MODE AND LOCATION DECISIONS}

In this section we present an example of an application where the advanced Computer-Based Causal Network Elicitation Technique (CB-CNET) is used. This tool has been developed by Kusumastuti et al. (2011) to automate CNET. At the same time, CB-CNET includes some modifications of the above-described network structure and elicitation procedure. One notable modification is that variable lists are shown to respondents and they indicate their responses by selecting items from them. Furthermore, attributes are framed as instruments to achieve benefits. The dynamic nature of the interface enables us to ask different questions to respondents depending on their previous selections. This procedure advances our knowledge about the associations between contexts, instruments and benefits.

Hasselt, a typical European historic city in Belgium, was chosen as a case study to implement the interface to assess the individuals' travel behaviour when engaging in leisure-shopping activities based on the CB-CNET interview, focusing on the transport mode and location choices. 221 respondents were recruited from $3-10 \mathrm{~km}$ away from the city centre. All respondents own a bike and have a driving licence. They also all have a bus stop within walking distance of their home (this applies for all people living near Hasselt due to its strong public transport policy). Accordingly, all respondents can consider each of three predefined transport modes (i.e. car, bus, and bike). The location context of the choice problem is further elucidated by showing a map of Hasselt city centre divided into three zones, derived from results of a preliminary study on participants' mental map. The three zones are: (1) the main shopping street, (2) an expensive boutique area, and (3) a gallery area.

To complete the research setting and scenario, the respondents are asked to reflect on two decisions: how to get to the city centre (transport mode decision) and what part of the centre to visit (shopping location decision). Following this step, all decision alternatives are explicated. The transport modes are explained by reminding the respondents that they are living on Hasselt outskirts and have various options to travel.

After describing the research setting and scenario, the elicitation procedure begins. In total, there are 27 contextual variables for the transport decision and 16 for the location decisions. The variables include natural conditions (e.g. weather conditions, wind, etc.), Travel Demand Management measures (e.g. bus frequency, parking cost, bus fare, etc.), and other contexts and constraints (time availability, parking availability, 
etc.). These predefined variables are developed based on literature and preliminary studies using the CNET interviews (Kusumastuti et al., 2011). To ensure that all respondents have uniform interpretations of the variables, the definition of each variable is shown in the interface whenever respondents pass the mouse on one of them.

Afterwards, respondents are asked how the benefits for each context are interconnected. For this purpose, 15 benefits are shown in a predefined benefit list (e.g. having fun, physical comfort, etc.). Next, the full cognitive subsets are elicited by asking the relevant attributes for each selected context-benefit. The interface automatically generates questions based on respondents' previous variable selections. After this procedure is completed, the first cognitive subset type (context-benefit-attribute) is registered. When a respondent initially points out that he or she would always directly choose a certain transport mode regardless of a specific context, another elicitation path is generated to obtain the generalized representations from needs (Wyer, 2007). The procedure begins with extracting all pursued benefits from the chosen transport mode, followed by revealing the linked instruments. The full list of attributes contains 25 variables for the transport choices and 23 for the location choices respectively. As a result, the second cognitive subset type ("always choose"-benefit-attribute) can be recorded. Additionally, participants are asked if they have considerations that are not represented in the lists. Figure 5.2 provides an example of the type of mental representation that can be derived with CB-CNET.

Probabilities are assessed based on the relationships between parent and child nodes. Theoretically, these probabilities should be gathered for each node but this is not feasible in practice, considering the amount of questions that respondents have to answer. Therefore, some assumptions are made. First, probabilities of certain contexts are assessed based on an individual's own beliefs. Next, the probabilities of instruments rely on the context states. The mentioned considerations let us focus solely on the probabilities of benefits, based on contexts and decisions. Utility weights are derived from two measures obtained as (1) importance ratings of single benefits and (2) ratings of combined benefits in a conjoint experiment using fractional factorial design profiles. For the importance ratings participants indicate the importance of gaining benefits when they go shopping. Respondents indicate their answers on continuous response bars, ranging from not important (0) to extremely important (100). Each value is divided by the sum of the elicited benefit values, yielding a set of utility weights. Note that alternative approaches are possible here to elicit benefit importance. In particular, recently researchers have introduced approaches based on best-worst measurement techniques that 


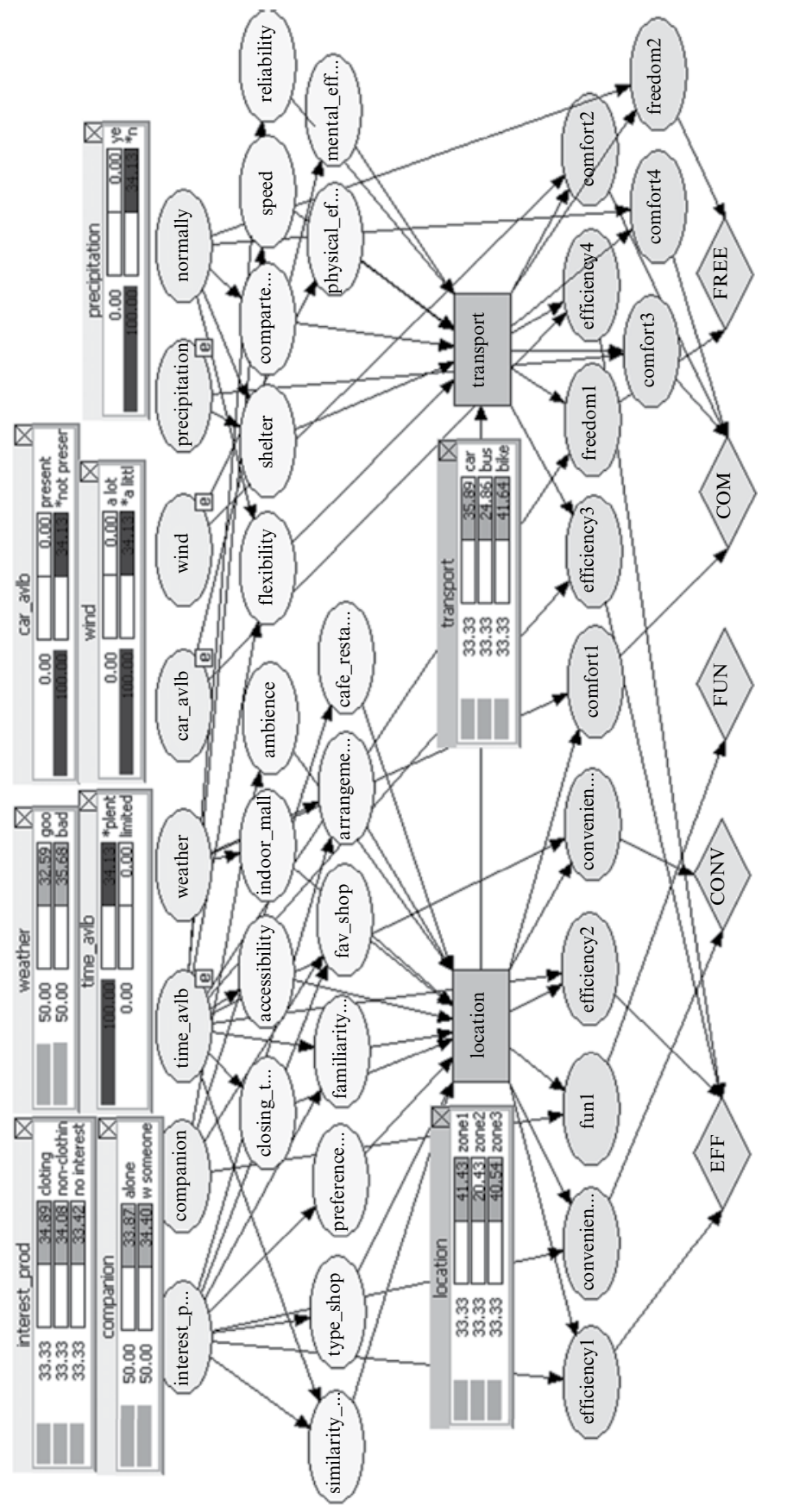

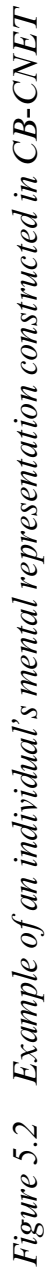


provide better possibilities to disentangle benefit importance and benefit evaluation (Louviere and Islam 2008). The conjoint experiment offers a second way to derive utility estimates that may circumvent this problem. This experiment uses fractional factorial designs to derive partial-utility weights. The respondents are asked to value the profiles separately concerning their chance to execute the leisure-shopping activity (0-100 per cent). Each profile contains combinations of the seven most important benefits for each participant. These benefits are taken from the survey. Thus, the same experimental design is used for all participants, but the assessed benefits differ from one respondent to another. Alternatively, discrete choice experiments can be used to measure benefit evaluations per respondent (e.g. Louviere et al. 2000).

The last part of the survey is designed to gather sufficient data for model validations. Respondents' actual transport mode preferences are asked according to the individuals' initial selected contexts. Ultimately, these preferences are compared with the DN results to check the model accuracy.

Some follow-up analyses on the Bayesian DN models derived from the data of the respondents were conducted to calculate the predictive accuracy of the models. Participants' actual transport mode choices in different scenarios as reported in the CB-CNET survey data are used as a benchmark to calculate how well the DN models predict the choices indicated by participants. Results show that the accuracy of the DN models is around 67 per cent, implying that in 67 per cent of the cases these models can correctly predict participants' transport mode and location choices under various contexts and constraints.

In general, the proposed automated approach significantly reduces the time to collect the data in comparison to the face-to-face CNET interview protocol because the parameters are automatically and directly gathered during the survey. On average, the whole survey lasted for about 2-3 hours, and was run in group sessions of about 20 respondents. This is considerably more efficient than the traditional separate face-to-face CNET interview.

\section{ADVANTAGES AND CHALLENGES OF MR BASED MODELS}

The MR approach has several advantages over traditional choice modelling approaches but also has its limitations. The same holds when comparing MR with the means-end based approach (Gutman 1982), which partly preceded the MR method. 
A main advantage of MR approaches is that the postulated model allows better representing (mimicking) the decision process that underlies the observed decision outcome. Stated and revealed preference methods (SP/RP) essentially model relationships between stimulus variations and variations in continuous or discrete outcomes (ratings or choices) based on assumptions of random utility theory. Context variables, whether decision maker or task characteristics, are included as moderators (or interactions), which allows developing models for predictive purposes and hypothesis testing. These models in the end however only capture context effects in statistical terms; they do not represent a structural explanation as to why the effects occur or should occur. Dividing respondents in (latent) classes based on differences in attribute preferences comes closer but still does not provide for different mental structures. The choice models that go furthest in representing such different structures are attempts to model variations in decision strategies (e.g., Swait and Adamowicz 2001). Similarly, the inclusion of process variables (mediators) in choice models only happens by exception, for example, when variables are included related to consideration or choice set construction (e.g., Cascetta and Pappola 2001; Swait and Ben-Akiva 1987) or hierarchies of perceptions (e.g., Oppewal et al. 1994; Timmermans and Molin 2009), again demonstrating the limited representational ability of these models. The MR models allow much greater flexibility regarding the representation of process variables because they explicitly include the perceived benefits of attributes and alternatives and imply a decision making process that is explicitly based on attribute salience driven by benefits relevant to a particular situation. This in turn allows insight into which benefits play an active role in a given situation. Practically this means that respondents can be grouped (segmented) on benefits sought instead of on benefits delivered (as in traditional attribute based benefit segmentation).

Additional appeal when considering MR versus traditional attribute based models used in SP/RP methods comes from MR methods explicitly aiming for the modelling of mental processes and the building of a representation that resembles the decision maker's view of the world. RP methods merely use observed outcomes and are therefore limited to existing, already experienced choice situations. SP methods probe respondents to consider alternative choice situations and express their preferences but by their nature depend on the respondent's ability to mentally simulate the presented decision situation and produce a response 'as if' the presented choice situation (decision scenario) were real. This is why attribute development and pretesting stages are crucial to the success of SP methods. Somewhat surprisingly the attention in the literature to this aspect of SP design is limited compared to focus on statistical design issues. In contrast, in the MR approach the explicit focus is on capturing the key features of 
the decision maker's mental model and on capturing the decision maker's view of the decision problem. Hence, MR methods in principle better equip the researcher to achieve 'understanding' of the decision process and predict the resulting behaviour in a particular context or setting.

When comparing the MR methods with their earlier cousin, meansend analysis, they firstly share the advantage, discussed above, that the postulated model has higher representational value of the perceptual and decision process that underlies the observed decision outcome than typical choice models. A limitation of MR methods however is that they are still inherently 'cognitive' and, while allowing for context-based differences in perceived benefits, they do not strictly include the motivational processes and drivers themselves. In contrast, the means-end approach (Gutman, 1982) explicitly assumes that behaviour is value based and that the decision maker's values lead the individual to seeking for and perceiving benefits in particular attributes.

A further limitation of all approaches discussed is their neglect of emotions. Whereas means-end approaches better than MR incorporate the value component of motivations, neither of them includes or addresses the role of emotions. The approaches share with choice modelling a bounded rationality perspective of decision makers acting upon their (value-based) beliefs. The MR similarly ignores emotions however because the approach in principle 'just maps out' which benefits are salient in which situation; it could allow including effects of emotions, although this seems not to have been attempted yet. It would be relevant to consider the role of 'hot' cognition and how affect influences mental representations (e.g., Loewenstein 1996; Wendel and Dellaert 2009).

Another challenge for the MR methods concerns the possible role of demand effects. The approach rightfully presents decision makers as active benefit seekers, not merely as passive benefit recognizers. This however means that when considering a choice situation, respondents are not only seeking to find out about the benefits of attributes, as assumed in the interview protocols. They may also wonder what the task itself is about, why those questions are being asked of them, whether they will be later queried to explain their answers, what it is they are supposed to be looking for, and also 'what's in it for them'. This means that issues need to be considered regarding social desirability and policy influencing attempts as well as, more in general, social and self justification effects (Bettman et al. 1998) on MR responses, whether there are missing or 'irrelevant' attributes that may lead the respondent to infer attributes and benefits (Carpenter et al. 1994), and also about the possible role of communication norms regarding what an interview 'should' look like (Xu and Wyer 2010). These issues also apply in stated preference settings (although not 
often recognized as such) but they can be expected to play a larger role in MR methods, which 'delve deeper' and hence ask the respondent to explore more of or about themselves. Note these same issues apply for the means-end approach and the laddering method, especially the original 'soft laddering' approach (Russell et al. 2004). One of the promises of implementations of the approach through online, non-personal interviews is that they may reduce these demand effects.

\section{FUTURE RESEARCH}

Before the many promises associated with capturing MRs in choice models can become reality, a number of challenges need to be met. Just as MRs are often used to try and capture the 'why' and 'how' of decision making, these challenges can be categorized into 'why' and 'how' questions.

To start with the 'why': MR researchers could highlight more explicitly than is currently being done, what is the added value of their research for researchers and practitioners. It is important to show empirically that MR models lead to more insight in behaviour and as such to more informed planning and policy making. It would be especially helpful if it could be shown how MR models lead to insights that are truly new, in the sense that they go beyond or even run counter to intuition. Similarly, it would be interesting to see how MR models - by providing more information than conventional choice models - might actually lead to different policies or marketing actions. Related to the notions of new behavioural insights and more informed policy making, are the issues of model fit and predictive validity of MR models. Although recent research has shown that MR models have the potential to outperform conventional choice models along these dimensions (Arentze et al. 2008; Dellaert et al. 2008), it should be noted that quite substantial gains in fit and predictive ability are in fact needed to offset the substantial losses in degrees of freedom and the nontrivial increases in time, effort and money associated with building and estimating MR models. As a 'new kid on the block', MR models have to show that incorporating MRs in choice models is literally worth the effort.

Furthermore, generalizability of MRs is an issue that needs attention. As the cognitive representations are individual-specific complex network structures the question arises how one can generalize behaviour based on MR data obtained from a sample. Two possible avenues are worth (further) exploring. The first is to develop a method to model the formation of MRs for different choice problems as a function of situational factors (context and decision maker variables). The activation of benefits and the strength of causal relationships that lead to subsequent activation 
of attributes possibly could be modelled drawing on theory costly information search that guides the selection of nodes and links in an MR. When estimated on collected MR data, a model of MR-formation would provide a generalization of the mental representations. Second, cluster or latent class analyses offer possible ways to derive taxonomies of MRs (for a given choice problem) that provide insights into generic characteristics of MRs. By simultaneous estimation of cluster- or class-membership functions, these can be regressed to variables related to the individual and context to obtain insights as to which type of MRs are activated under which conditions. In the context of both of these alternative approaches - theory-based model or cluster- or latent class-based analysis - the generic features that emerge can be related to choice behaviour of individuals when data on the choice context and individual is also recorded.

Moving to the 'how' question: although over the past few years a number of methodological advances have been made to increase the ease and reliability of MR-related data-collection and analysis, a number of methodological issues remain to be tackled. The most important of these relates perhaps to the empirically well established notion that asking individuals why they choose a particular alternative or how they arrived at their decision often leads to quite unreliable 'stories' (e.g., Nisbett and Wilson 1977). For example, Mueller et al. (2010) report a case where individuals were unaware of key factors impacting their choices. Systematic research is needed to obtain a clear indication on the extent to which responses such as obtained by the CNET elicitation method have predictive value for choice behaviour. Although it is clear that verbal reports are of crucial importance for the construction and estimation of MR models, the fact that these reports are known to be to some extent unreliable means that it would be worth investigating how they can be complemented by other evidence of decision making processes. Obvious candidates in this regard are methods like eye-tracking and neuro-imaging. Although these latter methodologies have been used extensively to study decision making (e.g., Coricelli et al. 2005), combining them with verbal reports to help construct and estimate MR-based choice models would be a new and promising approach. A related caveat would be that use of these methods would make MR models even more labour intensive than they already are without incorporating physiological or neuro-imaging evidence.

\section{CONCLUSION}

The premise of the study of mental representations (MRs) of decision makers is that the mental construction of a choice problem influences 
choice behaviour and, at the same time, is influenced by situational context. Many choices, such as travel or location choices be it for the short term (e.g., mode and destination of a trip) or longer term (e.g., transport mode availability, residential location) tend to be complex. They require simultaneous decisions on multiple dimensions and involve choice sets that have very many options and with outcomes that are hard to predict. The mental representation of such choice problems tends to involve strong reduction of reality in terms of attributes and benefits considered, implying that to understand choice behaviour it is important to know how the selective activation of these cognitions comes about. To date, econometric approaches have conceptualized and modelled heterogeneity in decision strategies in terms of taste variety, predominantly in mixed-logit and latent class frameworks (e.g., Train 2003). However, MRs, which may vary across individuals and situations, also affect the structure of evaluation functions and choice sets and may differ between individuals. The way decision makers construct reality, therefore, is an emerging field of research in behavioural decision making that holds the promise to enhance our understanding of choice behaviour and, in the end, explanatory and predictive ability of discrete choice models.

In this chapter, we reviewed basic concepts and methods to model and measure MRs and discussed potentials as well as challenges that still need to be addressed to further advance this area of research. The ambition is to formulate research approaches that go beyond traditional qualitative focus-groups and in-depth interviews that uncover key attributes for stated choice experiments and revealed discrete choice modelling. To date, progress has been made regarding questions such as: How can MRs of decision makers be conceptualized, modelled and measured? To what extent and in what ways do revealed MRs of travellers and consumers vary across situational contexts and individual characteristics? What is the influence of the MRs on choice behaviour and what are the implications for and prospect of incorporating this in discrete choice models? Although progress has been made, the research is still in an early stage and more definite answers have yet to be generated.

\section{REFERENCES}

Arentze, T.A., B.G.C., Dellaert, and H.J.P. Timmermans (2008), 'Modeling and Measuring Individuals' Mental Representations of Complex SpatioTemporal Decision Problems,' Environment and Behavior, 40 (November), 843-869.

Bettman, J.R., M.F. Luce, and J.W. Payne (1998), 'Constructive Consumer Choice Processes,' Journal of Consumer Research, 25 (Dec), 187-217. 
Carpenter, G.S, R. Glazer, and K. Nakamoto (1994), 'Meaningful Brands from Meaningless Differentiation: The Dependence on Irrelevant Attributes,' Journal of Marketing Research, 31 (August), 339-350.

Cascetta, E. and A. Papola (2001), 'Random Utility Models with Implicit Availability/Perception of Choice Alternatives for the Simulation of Travel Demand,' Transportation Research Part C: Emerging Technologies, 9 (4), 249-263.

Christensen, G.L., and J.C. Olsen (2002), 'Mapping Consumers' Mental Models with ZMET,' Psychology \& Marketing, 19, 477-502.

Coricelli, G., H.D. Critchley, M. Joffily, J.P. O’Doherty, A. Sirigu, and R.J. Dolan (2005), 'Regret and Its Avoidance: A Neuroimaging Study of Choice Behaviour,' Nature Neuroscience, 8 (9), 1255-1262.

Dellaert, B.G.C., T.A. Arentze, and H.J.P. Timmermans (2008), 'Shopping Context and Consumers' Mental Representation of Complex Shopping Trip Decision Problems,' Journal of Retailing, 84, 219-232.

Eden, C., and F. Ackermann (1998), Making Strategy: The Journey of Strategic Management, London: Sage.

Gutman, J. (1982), 'A Means-End Chain Model Based on Consumer Categorization Processes,' Journal of Marketing, 46, 60-72.

Horeni, O., T.A. Arentze, B.G.C. Dellaert, and H.J.P. Timmermans (2010), 'CNET and APT - a comparison of two methods for measuring mental representations underlying activity-travel choices,' in General Proceedings of the 12th World Conference on Transport Research Society.

Johnson-Laird, P.N. (1983), Mental Models, Cambridge, MA: Harvard University Press.

Johnson-Laird, P.N. (2001), 'Mental Models and Deduction,' Trends in Cognitive Sciences, 5 (10), 434-442.

Johnson-Laird, P.N. and R.M.J. Byrne (1991), Deduction, Hillsdale, NJ: Lawrence Erlbaum.

Kearney, A.R., and S. Kaplan (1997), 'Toward a Methodology for the Measurement of Knowledge Structures of Ordinary People: The Conceptual Content Cognitive Map (3CM),' Environment and Behavior, 29, 579-617.

Kusumastuti, D., E. Hannes, D. Janssens, G. Wets, and B.G.C. Dellaert (2010), 'Scrutinizing Individuals' Leisure-Shopping Travel Decisions to Appraise Activity-Based Models of Travel Demand,' Transportation, 37 (4), 647-661.

Kusumastuti, D., E. Hannes, B. Depaire, K. Vanhoof, D. Janssens, G. Wets, and B.G.C. Dellaert (2011), 'An Interactive Computer-Based Interface to Support the Discovery of Individuals' Mental Representations and Preferences in Decisions Problems: An Application to Travel Behavior,' Computers in Human Behavior, 27 (2), 997-1011.

Loewenstein, G. (1996), 'Out of Control: Visceral Influences on Behavior,' Organizational Behavior and Human Decision Processes, 65, 272-292.

Louviere, J.J. and T. Islam (2008), 'A Comparison of Importance Weights and Willingness-to-Pay Measures Derived from Choice-Based Conjoint, Constant Sum Scales and Best-Worst Scaling,' Journal of Business Research, 61, 903-911.

Louviere, J.J., D.A. Hensher and J.D. Swait (2000), Stated Choice Methods, Cambridge, UK: Cambridge University Press.

Mueller, S., L. Lockshin and J.J. Louviere (2010), 'What You See May Not Be What You Get: Asking Consumers What Matters May Not Reflect What They Choose,' Marketing Letters, 21, 335-350. 
Neapolitan, R.E. (1990), Probabilistic Reasoning in Expert Systems: Theory and Algorithms, New York: John Wiley.

Nisbett, R.E., and T.D. Wilson (1977), 'Telling More Than We Can Know: Verbal Reports on Mental Processes,' Psychological Review, 84, 231-259.

Oppewal, H., J.J. Louviere, and H.J.P. Timmermans (1994), 'Modeling Hierarchical Conjoint Processes with Integrated Choice Experiments,' Journal of Marketing Research, 31, 92-105.

Pearl, J. (1988), Probabilistic Reasoning in Intelligent Systems: Networks of Plausible Inference, San Francisco: Morgan Kaufman.

Reynolds, T. and J. Gutman (1988), 'Laddering Theory, Method, Analysis, and Interpretation,' Journal of Advertising Research, 28 (1), 11-29.

Russell, C.G., A. Busson, I. Flight, J. Bryan, J.A. van Lawick van Pabst, and D.N. Cox (2004), 'A Comparison of Three Laddering Techniques Applied to an Example of Complex Food Choice,' Food Quality and Preference, 15, 569-583.

Schachter, R. (1986), 'Evaluating Influence Diagrams,' Operations Research, 34, 871-882.

Spiegelhalter, D.J., A. Philip, S.L. Lauritzen, and R.G. Cowell (1993), 'Bayesian Analysis in Expert Systems,' Statistical Science, 8, 219-283.

Swait, J., and W. Adamowicz (2001), 'The Influence of Task Complexity on Consumer Choice: A Latent Class Model of Decision Strategy Switching,' Journal of Consumer Research, 28 (June), 135-148.

Swait, J. and M. Ben-Akiva (1987), 'Incorporating Random Constraints in Discrete Choice Models of Choice Set Generation,' Transportation Research, 21B, 91-102.

Timmermans, H.J.P. and E.J.E. Molin (2009), 'Dealing with Increased Complexity in Conjoint Experiments: Background and Overview of Alternate Approaches,' Transport Reviews, 29 (5), 557-567.

Train, K. (2003), Discrete Choice Methods with Simulation, New York: Cambridge University Press.

Wendel, S. and B.G.C. Dellaert (2009), 'Situation-Based Shifts in Consumer Website Benefit Importance: The Joint Role of Cognition and Affect,' Information \& Management, 46 (1), 23-30.

Wyer, R. (2007), 'Principles of Mental Representation,' In A.W. Kruglanski and E.T. Higgins (eds), Social Psychology, Second Edition: Handbook of Basic Principles (pp. 285-307). New York: The Guilford Press.

$\mathrm{Xu}$, A.J. and R.S. Wyer (2010), 'Puffery in Advertisements: The Effects of Media Context, Communication Norms, and Consumer Knowledge,' Journal of Consumer Research, 37 (August), 329-342. 